\title{
Implementation and Comparison of Acoustic Travel-Time Measurement Procedures for the Solar Dynamics Observatory/Helioseismic and Magnetic Imager Time - Distance Helioseismology Pipeline
}

\author{
S. Couvidat · J. Zhao • A.C. Birch • A.G. Kosovichev • \\ T.L. Duvall Jr. • K. Parchevsky • P.H. Scherrer
}

Received: 28 December 2009 / Accepted: 29 September 2010 / Published online: 4 November 2010

(C) The Author(s) 2010. This article is published with open access at Springerlink.com

\begin{abstract}
The Helioseismic and Magnetic Imager (HMI) instrument onboard the Solar Dynamics Observatory (SDO) satellite is designed to produce high-resolution Doppler-velocity maps of oscillations at the solar surface with high temporal cadence. To take advantage of these high-quality oscillation data, a time-distance helioseismology pipeline (Zhao et al., Solar Phys. submitted, 2010) has been implemented at the Joint Science Operations Center (JSOC) at Stanford University. The aim of this pipeline is to generate maps of acoustic travel times from oscillations on the solar surface, and to infer subsurface 3D flow velocities and sound-speed perturbations. The wave travel times are measured from cross-covariances of the observed solar oscillation signals. For implementation into the pipeline we have investigated three different travel-time definitions developed in time-distance helioseismology: a Gabor-wavelet fitting (Kosovichev and Duvall, SCORE'96: Solar Convection and Oscillations and Their Relationship, ASSL, Dordrecht, 241, 1997), a minimization relative to a reference cross-covariance function (Gizon and Birch, Astrophys. J. 571, 966, 2002), and a linearized version of the minimization method (Gizon and Birch, Astrophys. J. 614, 472, 2004). Using Doppler-velocity data from the Michelson Doppler Imager (MDI) instrument onboard SOHO, we tested and compared these definitions for the mean and difference traveltime perturbations measured from reciprocal signals. Although all three procedures return similar travel times in a quiet-Sun region, the method of Gizon and Birch (Astrophys. J. 614, $472,2004)$ gives travel times that are significantly different from the others in a magnetic (active) region. Thus, for the pipeline implementation we chose the procedures of Koso-
\end{abstract}

The Solar Dynamics Observatory

Guest Editors: W. Dean Pesnell, Phillip C. Chamberlin, and Barbara J. Thompson.

S. Couvidat $(\bowtie) \cdot$ J. Zhao $\cdot$ A.G. Kosovichev $\cdot$ K. Parchevsky $\cdot$ P.H. Scherrer

W.W. Hansen Experimental Physics Laboratory, Stanford University, 491 S Service Road, Stanford, CA 94305-4085, USA

e-mail: couvidat@stanford.edu

A.C. Birch

CoRA Division, NorthWest Research Associates, 3380 Mitchell Lane, Boulder, CO 80301, USA

T.L. Duvall Jr.

Solar Physics Laboratory, NASA/GSFC, Greenbelt, MD 20771, USA 
vichev and Duvall (SCORE'96: Solar Convection and Oscillations and Their Relationship, ASSL, Dordrecht, 241, 1997) and Gizon and Birch (Astrophys. J. 571, 966, 2002). We investigated the relationships among these three travel-time definitions, their sensitivities to fitting parameters, and estimated the random errors that they produce.

Keywords Sun: helioseismology $\cdot$ Sun: time-distance analysis $\cdot$ HMI

\section{Introduction}

Time-distance helioseismology (Duvall et al., 1993) allows access to the subsurface physical properties of the quiet Sun (see e.g. Kosovichev and Duvall, 1997), including supergranulation (see e.g. Duvall and Gizon, 2000; Zhao and Kosovichev, 2003; Hirzberger et al., 2007), and also the subsurface properties of active regions (see e.g. Kosovichev, Duvall, and Scherrer, 2000; Zhao, Kosovichev, and Duvall, 2001; Jensen et al., 2001; Couvidat, Birch, and Kosovichev, 2006). So far the main goal has been to determine the velocity of material flows in both the horizontal and vertical directions, and the sound-speed profile as a function of depth. As such, time-distance helioseismology is a major tool to achieve the primary science goal of the Helioseismic and Magnetic Imager (HMI) instrument onboard the Solar Dynamics Observatory (SDO) satellite: to study the origin of solar variability, and to characterize and understand the Sun's interior and various components of magnetic activity. Therefore, a time-distance helioseismology data-analysis pipeline (described by Zhao et al., 2010) has been implemented by the authors, at the SDO Joint Science Operations Center (JSOC). It will process the line-of-sight Doppler-velocity (and potentially continuum intensity) maps obtained by HMI to generate wave travel-time maps of the solar surface and invert these maps to generate estimates of the flow velocities and sound-speed perturbations beneath the surface.

The travel times of acoustic or surface-gravity wavepackets are measured from temporal cross-covariances of solar-oscillation signals measured at different positions on the solar disk. Over the years, various definitions of these travel times have been designed (Kosovichev and Duvall, 1997 [hereafter KD97]; Gizon and Birch, 2002 [hereafter GB02]; Gizon and Birch, 2004 [hereafter GB04]). They generally return different times for a given cross-covariance function. Upon their implementation in the HMI time-distance pipeline we compare the results of these definitions using Doppler-velocity data from the Michelson Doppler Imager (MDI) instrument (Scherrer et al., 1995). We compare these three traveltime definitions for both quiet-Sun and active-region data. We only compute the mean and difference travel-time perturbations in the center-to-annulus geometric scheme, neglecting the North - South and East - West travel-time differences (see Couvidat and Birch, 2009, for a comparison of these travel-time differences in the presence of artificial uniform and steady flows, and for the three travel-time definitions). Moreover, we only consider acoustic waves ( $p$ modes). Jackiewicz et al. (2007) already studied the travel times of surface-gravity waves ( $f$ modes). Finally, Roth, Gizon, and Beck (2007) compared the travel-time differences returned by the KD97 and GB04 methods in the quiet Sun. In this paper we expand part of their analysis to include the GB02 approach, to include mean travel-time perturbations, and we also work on active-region data. Other possible ways to measure the travel-time shifts of wavepackets are not considered here. For instance, it is possible to compute the instantaneous phase of the cross-covariance using the Hilbert transform, and to define the travel time as the time of zero-crossing of this instantaneous phase (see e.g. Duvall et al., 1996). Other related techniques are phase-correlation holography (Lindsey and Braun, 2005) and acoustic imaging (Chou and Duvall, 2000). 
In Section 2 we review the time-distance formalism and discuss the travel-time definitions. In Section 3 we present the implementation of these travel-time measurement procedures in the HMI JSOC data-analysis pipeline. In Section 4 we compare the results of these different approaches for quiet-Sun and active-region data from SOHO/MDI. Finally, we present our conclusions in Section 5.

\section{Time-Distance Formalism}

\subsection{Computation of the Cross-Covariances of Solar Oscillations}

In this section we outline the cross-covariance calculations used for testing our travel-time measurement procedures and codes. We apply the measurement procedure detailed by Couvidat, Birch, and Kosovichev (2006). The measurements of the travel times are based on fitting the cross-covariances $\left[C\left(\mathbf{r}_{1}, \mathbf{r}_{2}, t\right)\right]$ between source $\left[\mathbf{r}_{1}\right]$ and receiver $\left[\mathbf{r}_{2}\right]$ calculated from a line-of-sight-velocity data cube $[\phi(\mathbf{r}, t)]$ where $\mathbf{r}$ is the horizontal position vector, and $t$ is time:

$$
C\left(\mathbf{r}_{1}, \mathbf{r}_{2}, t\right)=\frac{1}{T} \int_{0}^{T} \phi\left(\mathbf{r}_{1}, t^{\prime}\right) \phi\left(\mathbf{r}_{2}, t^{\prime}+t\right) \mathrm{d} t^{\prime}
$$

Here $T$ is the temporal duration of the observation. A high-pass filter is applied at $1.7 \mathrm{mHz}$ to the Fourier transform of the data cube, to discard supergranulation. An $f$-mode filter is applied to discard the $f$-mode signal. To reduce the noise level of the cross-covariances, $\phi(\mathbf{r}, t)$ is filtered in the Fourier domain using a Gaussian phase-speed filter (Duvall et al., 1997) for short travel distance $\Delta=\left|\mathbf{r}_{2}-\mathbf{r}_{1}\right|$. Then, the point-to-point cross-covariances are averaged over annuli of radius $\Delta$ centered on the source. Such point-to-annulus crosscovariances are computed for 55 distances $[\Delta]$ ranging from 3.7 Mm to $66.7 \mathrm{Mm}$. Only 11 phase-speed filters are applied for these 55 distances (one filter for five distances). These distances are not those selected for the time-distance pipeline: they are used here only to test our implementation of the travel-time definitions. To further increase the signal-to-noise ratio for the point-to-annulus cross-covariances, the latter are shifted in time - so that they are centered on specific reference times - and averaged in groups of five distances (the five $\Delta$ computed with the same phase-speed filter), thus giving only 11 final $\Delta$ values for measuring the acoustic travel times. For instance, five cross-covariances computed at distances ranging from 3.7 to $8.7 \mathrm{Mm}$ are shifted in time to be centered on the same reference time (here the group travel time of a wavepacket propagating in the quiet Sun over the distance $\Delta=6.2 \mathrm{Mm}$, as measured by the Gabor-wavelet fit presented in the next section) and are averaged to produce the final point-to-annulus cross-covariance at $\Delta=6.2 \mathrm{Mm}$. The values of $\Delta$ and properties of the phase-speed filters used in this paper are summarized in Table 1.

\subsection{Travel-Time Definitions Implemented in the Pipeline}

The travel times of the acoustic wavepackets are measured from the point-to-annulus crosscovariances. Historically, the cross-covariances were fitted by a Gabor wavelet defined as (Kosovichev and Duvall, 1997) [KD97]:

$$
G\left(A, \omega_{0}, \delta \omega, \tau_{\mathrm{p}}, \tau_{\mathrm{g}} ; t\right)=A \cos \left(\omega_{0}\left(t-\tau_{\mathrm{p}}\right)\right) \exp \left(-\frac{\delta \omega^{2}}{4}\left(t-\tau_{\mathrm{g}}\right)^{2}\right)
$$


Table 1 Parameters used for the time-distance analysis: central source-receiver distances $[\Delta]$, range of $\Delta$, properties of the Gaussian phase-speed filters applied to the Fourier transform of the line-of-sight-velocity data cube ( $v_{0}$ is the central phase speed and FWHM is the full width at half maximum), and centers $\left[t_{0}\right]$ of the temporal window applied to isolate the first-bounce skip on the cross-covariances.

\begin{tabular}{rcccc}
\hline$\Delta(\mathrm{Mm})$ & range $(\mathrm{Mm})$ & $v_{0}\left(\mathrm{~km} \mathrm{~s}^{-1}\right)$ & FWHM $\left(\mathrm{km} \mathrm{s}^{-1}\right)$ & $t_{0}(\mathrm{~min})$ \\
\hline 6.20 & $3.70-8.70$ & 12.77 & 6.18 & 19.0 \\
8.70 & $6.20-11.2$ & 14.87 & 6.18 & 23.3 \\
11.60 & $8.70-14.5$ & 17.49 & 6.18 & 24.4 \\
16.95 & $14.5-19.4$ & 24.82 & 9.09 & 28.7 \\
24.35 & $19.4-29.3$ & 35.46 & 12.36 & 33.5 \\
30.55 & $26.0-35.1$ & 39.71 & 7.18 & 36.3 \\
36.75 & $31.8-41.7$ & 43.29 & 7.42 & 38.7 \\
42.95 & $38.4-47.5$ & 47.67 & 8.41 & 40.8 \\
49.15 & $44.2-54.1$ & 52.26 & 10.5 & 42.8 \\
55.35 & $50.8-59.9$ & 57.16 & 8.90 & 44.7 \\
61.65 & $56.6-66.7$ & 61.13 & 8.03 & 45.0 \\
\hline
\end{tabular}

where $A$ is the amplitude of the wavelet, $\omega_{0}$ is the central temporal frequency of the wavepacket, $\delta \omega$ is a measure of its frequency width, $\tau_{\mathrm{p}}$ is the phase-travel time, and $\tau_{\mathrm{g}}$ is the group travel time. Commonly, only phase-travel times are used for the time-distance inversions, because they can usually be determined more accurately than the group travel times. However, $\tau_{\mathrm{p}}$ is not unique and is defined modulo $2 \pi / \omega_{0}$. Therefore, the following rule has been used in this paper to select the phase-travel times: the value of $\tau_{\mathrm{p}}$ that is selected is the closest to - and if two are equally distant, smaller than - the group travel time $\tau_{\mathrm{g}}$.

Subsequently, two definitions of the wavepacket travel times based on studies in geophysics were added. Gizon and Birch (2002) [GB02] define the travel time $\tau(\mathbf{r}, \Delta)$ as:

$$
\tau_{ \pm}(\mathbf{r}, \Delta)=\underset{t}{\arg \min } X_{ \pm}(\mathbf{r}, \Delta, t)
$$

where $X_{ \pm}(\mathbf{r}, \Delta, t)$ are the functions:

$$
X_{ \pm}(\mathbf{r}, \Delta, t)=\sum_{t^{\prime}} f\left( \pm t^{\prime}\right)\left[C\left(\mathbf{r}, \Delta, t^{\prime}\right)-C^{\mathrm{ref}}\left(\Delta, t^{\prime} \mp t\right)\right]^{2}
$$

and $f(t)$ is a one-sided window function that selects the first-bounce skip of the crosscovariance (the skip corresponding to waves traveling directly from the center to the annulus) for the positive-time (i.e. outgoing waves) or negative-time (i.e. ingoing waves) branches of the cross-covariance. The same window function is used when fitting the cross-covariance by a Gabor wavelet even though it does not explicitly appear in Equation (2). The function $C^{\text {ref }}(\Delta, t)$ is a reference cross-covariance. Here, $C^{\text {ref }}(\Delta, t)$ is calculated as a spatial average of the measured cross-covariances over a region of the quiet Sun.

Lastly, Gizon and Birch (2004) [GB04] proposed another definition of the travel time that is linear with respect to the cross-covariance:

$$
\tau_{ \pm}(\mathbf{r}, \Delta)=\sum_{t} W_{ \pm}(\Delta, t)\left[C(\mathbf{r}, \Delta, t)-C^{\mathrm{ref}}(\Delta, t)\right]
$$


where $W_{ \pm}$is a weight function:

$$
W_{ \pm}(\Delta, t)=\frac{\mp f( \pm t) \dot{C}^{\mathrm{ref}}(\Delta, t)}{\sum_{t^{\prime}} f\left( \pm t^{\prime}\right)\left[\dot{C}^{\mathrm{ref}}\left(\Delta, t^{\prime}\right)\right]^{2}}
$$

where $\dot{C}^{\text {ref }}(\Delta, t)$ denotes the temporal derivative of the reference cross-covariance. Essentially, GB04 is a linearization of GB02 with respect to a parameter $\epsilon$ when $\epsilon \rightarrow 0$, where $\epsilon$ is used to define a smooth cross-covariance $C^{\epsilon}(\mathbf{r}, \Delta, t)$ :

$$
C^{\epsilon}(\mathbf{r}, \Delta, t)=\epsilon C(\mathbf{r}, \Delta, t)+(1-\epsilon) C^{\mathrm{ref}}(\Delta, t)
$$

The definition GB02 applied to $C^{\epsilon}(\mathbf{r}, \Delta, t)$ simplifies to GB04 in the limit $\epsilon \rightarrow 0$. For noiseless data, i.e. for an observation time $T=+\infty$, and when the amplitude of the crosscovariance does not vary, GB02 and GB04 are equivalent. GB04 can be applied only when the amplitude variations of $C(\mathbf{r}, \Delta, t)$ are small. This is not the case in active regions. While the travel-time variations in sunspots do not exceed, roughly, $20 \%$ of the typical wave period, the cross-covariance amplitude changes by more than 100\%. With GB04 there is a linear relationship between the travel times and $C(\mathbf{r}, \Delta, t)-C^{\text {ref }}(\Delta, t)$.

As emphasized by the use of the \pm symbol, for the three definitions of the travel times we measure $\tau$ separately for the outgoing $\left(\tau_{+}\right)$and ingoing $\left(\tau_{-}\right)$waves. We define the mean travel time, $\tau_{\text {mean }}(\mathbf{r}, \Delta)=\left(\tau_{+}(\mathbf{r}, \Delta)+\tau_{-}(\mathbf{r}, \Delta)\right) / 2$, and the difference travel time, $\tau_{\text {diff }}(\mathbf{r}, \Delta)=\tau_{+}(\mathbf{r}, \Delta)-\tau_{-}(\mathbf{r}, \Delta)$. The corresponding travel-time perturbations $\delta \tau$ are produced by subtracting the travel time obtained from the reference cross-covariance. For instance, $\delta \tau_{\text {mean }}(\mathbf{r}, \Delta)=\tau_{\text {mean }}(\mathbf{r}, \Delta)-\tau_{\text {mean; ref }}(\Delta)$ where $\tau_{\text {mean; ref }}(\Delta)$ is the mean travel time measured for the reference cross-covariance.

\section{Implementation of the Travel-Time Definitions in the HMI Time-Distance Pipeline}

The three travel-time definitions were implemented in the Fortran codes of the HMI time-distance helioseismology pipeline, based on the Data Resource Management System (DRMS) at Stanford University. They represent subroutines of the main time-distance program, which is also written in Fortran and encapsulated inside a $\mathrm{C}$-wrapper to access the DRMS I/O functions. The input parameters of the travel-time subroutines include the crosscovariances, and their output are the travel-time maps in FITS format. The mean and difference travel-time maps are calculated, as well as the North - South and East - West difference travel-time maps in a center-to-quadrant geometry (used to measure horizontal flows). The Gabor-wavelet fitting procedure (KD97) is a least-squares fit using the LevenbergMarquardt algorithm. The GB02 and GB04 procedures use a Whittaker-Shannon interpolation formula to interpolate the temporal cross-covariances from a grid with a 45 -second sampling rate (nominal HMI cadence) or a 60 -second sampling rate (MDI cadence) onto a grid with a five-second sampling rate. The interpolation subroutine computes interpolated points only in a narrow time range around the temporal window chosen to select the firstbounce skip (see, e.g., the last column of Table 1 for some typical values). This speeds up the computations.

For the GB02 definition, the code uses the interpolated cross-covariances to find the value of $t=t_{\min }$ at which $X_{ \pm}(\mathbf{r}, \Delta, t)$ is minimum. Following Gizon and Birch (2002) we then fit $X_{ \pm}(\mathbf{r}, \Delta, t)$ with a parabola around $t_{\min }$ (using five measurement points). The code 
returns an estimate of the parameters $c_{0}, c_{1}$, and $c_{2}$ of the parabola $y=c_{0}+c_{1} t+c_{2} t^{2}$, and the travel-time measurement is given by the location of the minimum of this parabola $\tau_{ \pm}=c_{1} /\left(2 c_{2}\right)$.

For the GB04 definition, the time derivative $\dot{C}^{\text {ref }}(\Delta, t)$ is numerically computed for the interpolated reference cross-covariance using a five-point equation.

The three travel-time definitions return outliers (misfits in the case of KD97 and GB02). To correct for these outliers, each travel-time subroutine computes the mean $[\mu]$ and standard deviation $[\sigma]$ of the ingoing and outgoing travel times. When $\left|\tau_{ \pm}(\mathbf{r}, \Delta)-\mu_{ \pm}\right|$is greater than $X$ times $\sigma_{ \pm}$(where $X$ is a parameter that can be set in the code; its value is typically larger than three), the cross-covariance at location $\mathbf{r}$ is averaged over the eight closest neighboring locations. Then the travel times at $\mathbf{r}$ are re-computed using the averaged crosscovariances.

\section{Comparison of the Travel-Time Definitions}

To test our implementation of the travel-time definitions, we apply them to MDI Dopplervelocity data. This allows a comparison of the travel-time definitions in the quiet Sun and in an active region.

\subsection{Quiet-Sun Region}

We work on a line-of-sight Doppler-velocity data cube obtained from MDI high-resolution (Hi-Res) data taken in May 2001 for a quiet-Sun region (Gizon and Birch, 2004). By quiet Sun we mean a region devoid of any significant magnetic activity (e.g., no sunspot, no plage). The observation duration is 512 minutes (or 8.5 hours). We choose the Hi-Res data because the spatial sampling rate $\left(0.6^{\prime \prime}\right.$ per pixel) is close to the HMI sampling rate. The data were remapped onto the heliographic coordinates using Postel's projection, and the region was tracked at the Carrington rate. After rebinning, the spatial resolution of our data cube is $\delta x=\delta y=0.826 \mathrm{Mm}$, and the temporal resolution is $\delta t=1$ minute. The dimensions of this data cube are $256 \times 256 \times 512$ where the first two dimensions are the horizontal ones, and the last dimension is the temporal one. We apply the time-distance formalism on this data cube. By default, the temporal windows used to select the first-bounce skip on the crosscovariances are 20 minutes wide. Figures 1 and 2 show the mean and difference travel-time perturbation maps obtained for two distances $\Delta=6.2$ and $\Delta=30.55 \mathrm{Mm}$ using the three travel-time definitions. All of the maps look very similar: they exhibit the same features at the same locations. However, the amplitude of these features varies. At $\Delta=6.2 \mathrm{Mm}$, the amplitude of $\delta \tau_{\text {diff }}(\mathbf{r}, \Delta)$ in the supergranules (black spots on the maps) is larger in absolute value for GB04 than for GB02 and KD97. Table 2 lists the values of the Pearson linear correlation coefficients between the different travel-time maps obtained for the quiet Sun. These coefficients are high, especially between GB02 and GB04.

Figure 3 shows histograms of the mean and difference travel-time perturbations for $\Delta=6.2$ and $\Delta=30.55 \mathrm{Mm}$. The travel-time distributions for the three definitions look very similar (more or less close to a Gaussian distribution). The standard deviations are larger for GB02 and GB04 than for KD97 at $\Delta=6.2 \mathrm{Mm}$, but they are smaller at $\Delta=30.55 \mathrm{Mm}$. Therefore, while GB02 and GB04 seem to produce slightly "noisier" travel-time maps at short distances than KD97, this is the opposite for larger distances (what matters is actually not the standard deviation - an assessment of the noise level - but the signal-to-noise ratio). This is consistent with the results of Roth, Gizon, and Beck (2007) for very large 

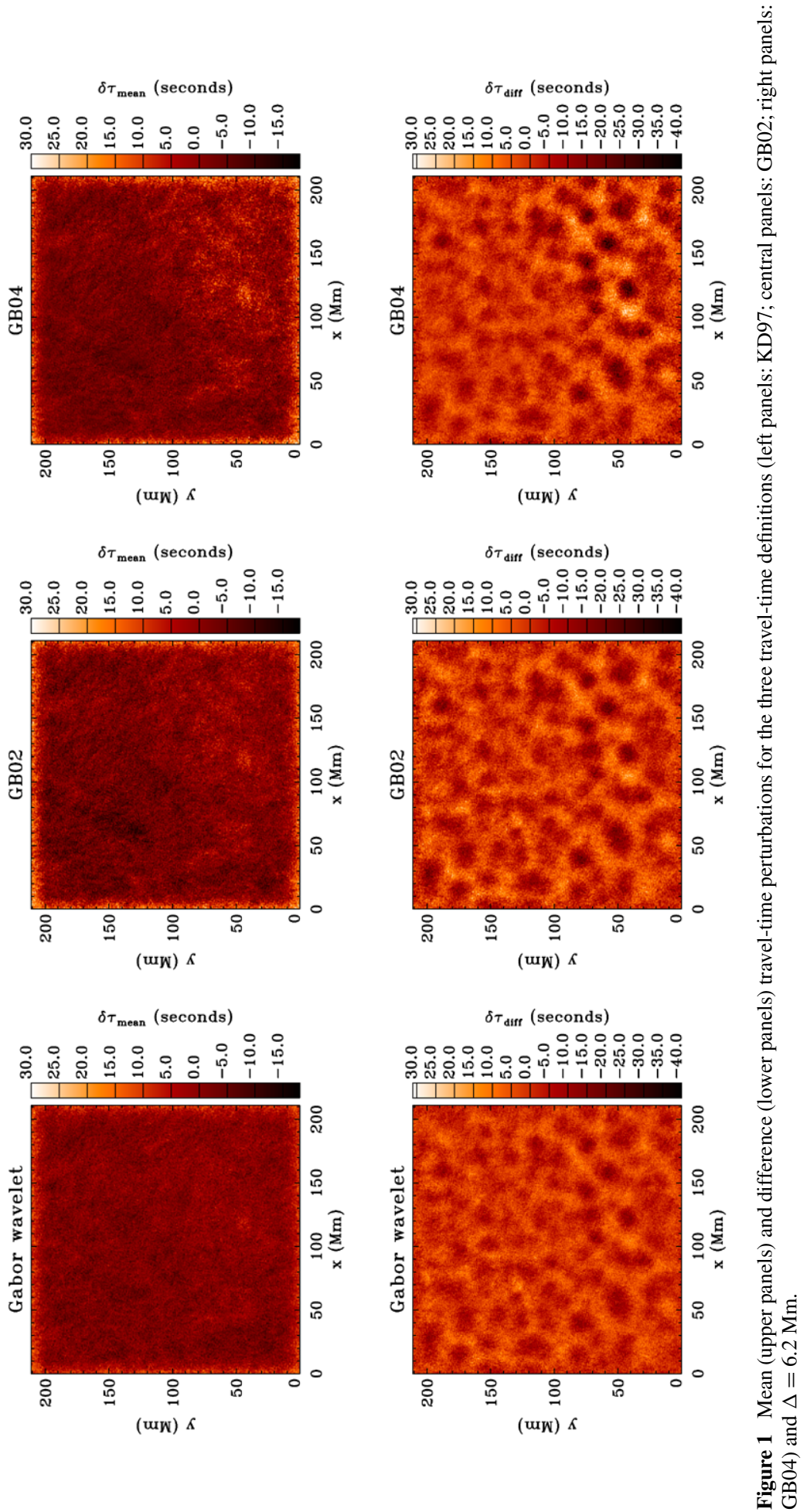


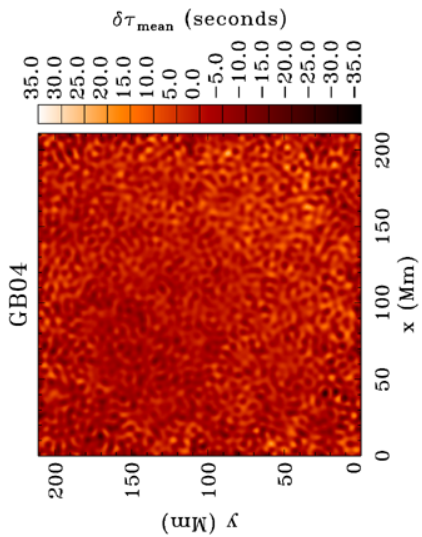

$$
\delta \tau_{\text {mean }} \text { (seconds) }
$$

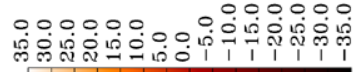

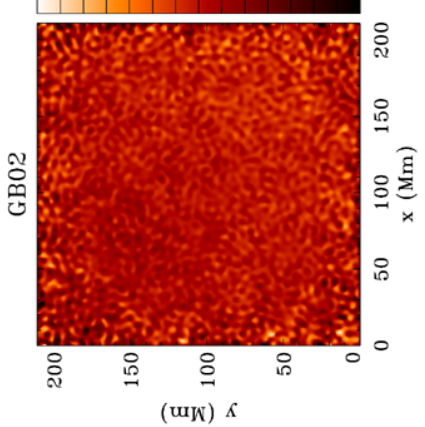

$\delta \tau_{\text {mean }}$ (seconds)

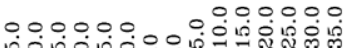
ம்

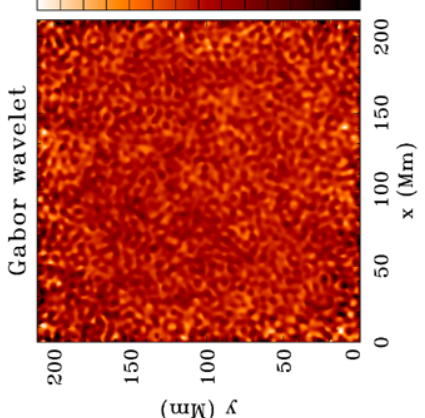

$\delta \tau_{\text {diff }}$ (seconds)

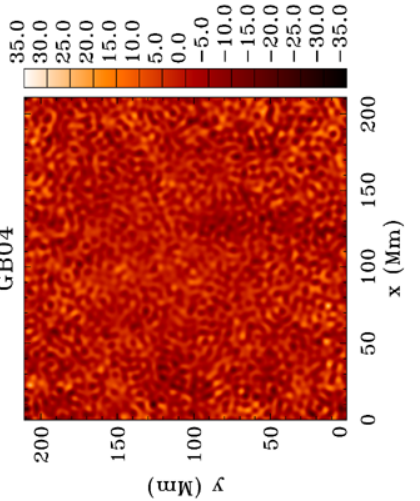

$\delta \tau_{\text {diff }}$ (seconds)

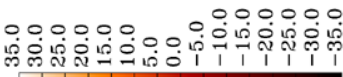

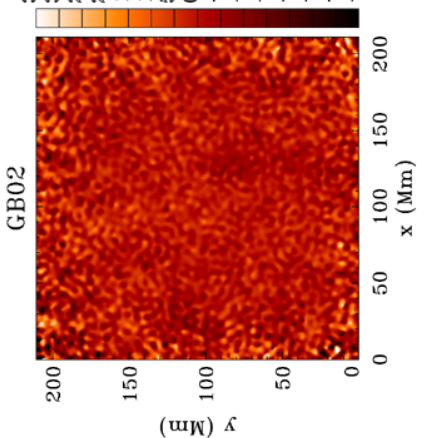

$\delta \tau_{\text {diff }}$ (seconds)

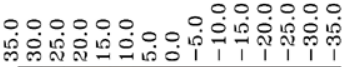

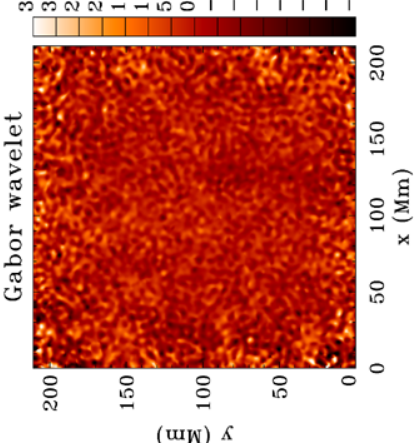

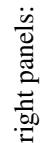

ญิ่

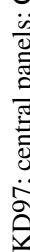

$\ddot{\ddot{m}}$

巳一ㄹㄹ

:

券

$\stackrel{\Xi}{\Xi}$

$\frac{1}{2} \cdot \Xi$

苛

ญ

$\pm$

$\Xi$

흠

ติ

灵

告

¿

\&

光

ले

$\pm 1$

我

퐁

¿)

总

¿

ठัญ

गे दे

늠

륭

สิ

के

ह

的

ङ?

छี

$\sum_{i}^{\infty} \triangleleft$

ㅜㅡㅇ

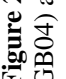


Table 2 Linear Pearson correlation coefficients between the different travel-time maps in the quiet Sun, for the entire $256 \times 256$ maps.

\begin{tabular}{llll}
\hline Map & KD97/GB02 & KD97/GB04 & GB02/GB04 \\
\hline$\delta \tau_{\text {mean }} \Delta=6.2 \mathrm{Mm}$ & 0.781 & 0.748 & 0.956 \\
$\delta \tau_{\text {mean }} \Delta=30.55 \mathrm{Mm}$ & 0.783 & 0.726 & 0.940 \\
$\delta \tau_{\text {diff }} \Delta=6.2 \mathrm{Mm}$ & 0.876 & 0.839 & 0.945 \\
$\delta \tau_{\text {diff }} \Delta=30.55 \mathrm{Mm}$ & 0.849 & 0.797 & 0.913 \\
\hline
\end{tabular}
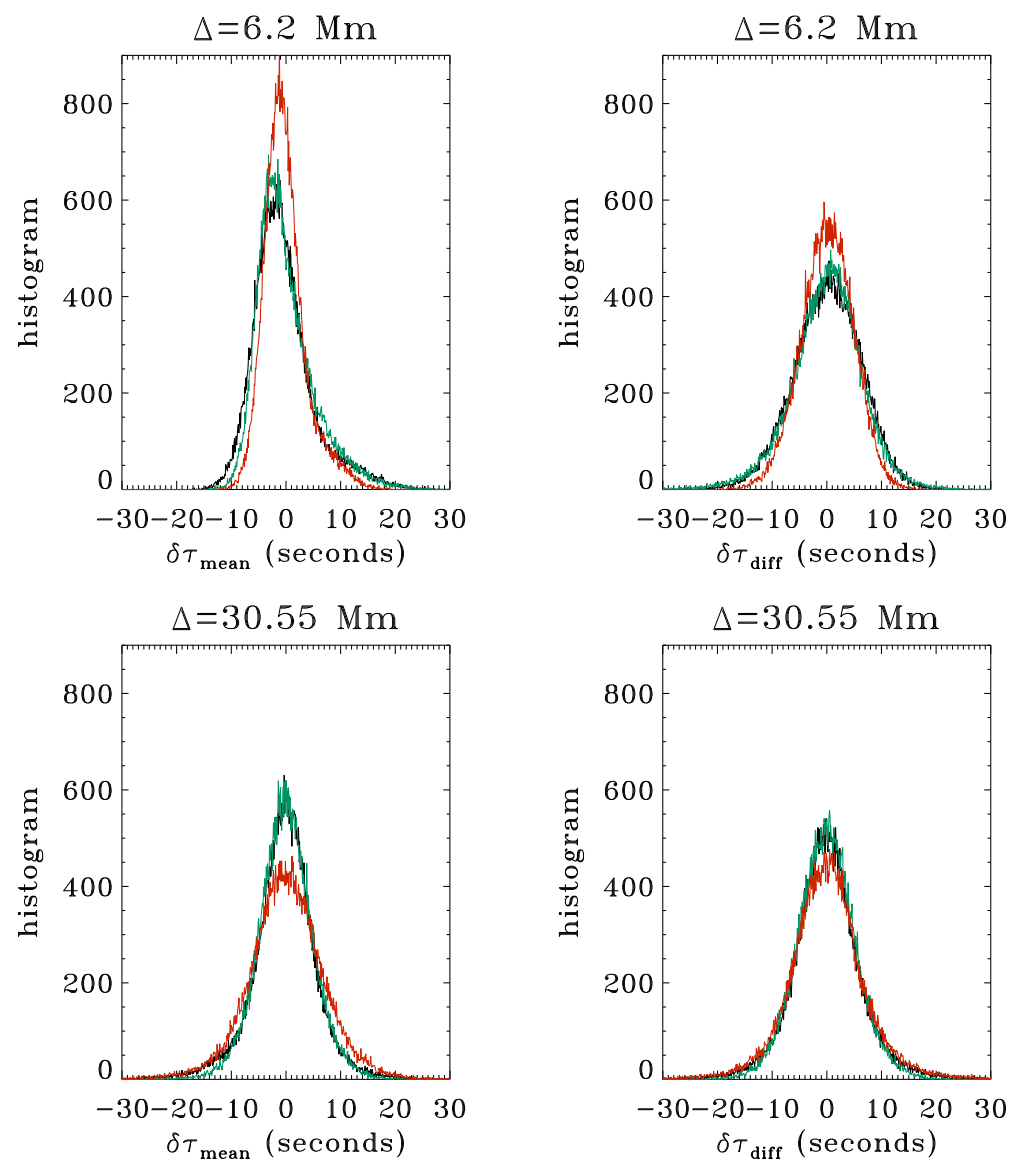

Figure 3 Histograms of the mean (left column) and difference (right column) travel-time perturbations for the two distances $\Delta=6.2$ (upper panels) and $\Delta=30.55$ (lower panels) Mm. Black lines are for GB02, green lines are for GB04, and red lines are for KD97.

distances (24 heliocentric degrees, or about $291 \mathrm{Mm}$ ). For short distance, here $\Delta=6.2 \mathrm{Mm}$, the distributions of the mean travel-time perturbations are noticeably different from a normal (Gaussian) distribution because of a tail at positive $\delta \tau_{\text {mean }}$. This is an edge effect: near the edges of the mean travel-time perturbation maps, $\delta \tau_{\text {mean }}$ is predominantly positive and large. Indeed, narrow white bands are clearly visible on these maps. These bands are produced by 
Table 3 Linear Pearson correlation coefficients between the different travel-time maps in the quiet Sun, for truncated maps: instead of using the full $256 \times 256$ pixels, we restrict the calculation to an inner square of $215 \times 215$ pixels, thus avoiding the map edges.

\begin{tabular}{llll}
\hline Map & KD97/GB02 & KD97/GB04 & GB02/GB04 \\
\hline$\delta \tau_{\text {mean }} \Delta=6.2 \mathrm{Mm}$ & 0.550 & 0.556 & 0.958 \\
$\delta \tau_{\text {mean }} \Delta=30.55 \mathrm{Mm}$ & 0.673 & 0.639 & 0.964 \\
$\delta \tau_{\text {diff }} \Delta=6.2 \mathrm{Mm}$ & 0.887 & 0.852 & 0.953 \\
$\delta \tau_{\text {diff }} \Delta=30.55 \mathrm{Mm}$ & 0.873 & 0.835 & 0.952 \\
\hline
\end{tabular}

the algorithm we use to compute the cross-covariances: the center-to-annulus averaging is performed in the Fourier domain by applying the convolution theorem. However, because the Doppler-velocity data cube is not periodic in the horizontal direction, this creates a spurious behavior at the edges of the map. Ignoring these map edges produces distributions that are very close to Gaussian ones. It also somewhat reduces the correlation coefficients KD97/GB02 and KD97/GB04 for the mean travel times, but not for the difference travel times (see Table 3). The correlation coefficients for the difference travel times remain high. At $\Delta=6.2 \mathrm{Mm}$, this is probably because the difference maps, unlike the mean travel-time maps, include a large-scale regular signal (supergranular flows) and not just noise (caused by convection and random realization of solar oscillations). At $\Delta=30.55 \mathrm{Mm}$, there is no real signal, and what we see on the maps is realization noise (or, more exactly, filtered realization noise, since the data cube was phase-speed filtered). Therefore at this distance we are testing how the different methods are measuring the noise.

We also tested the impact of the location and width of the temporal window on the mean and difference travel-time values. Figures 4, 5, and 6 show scatter plots for KD97, GB02, and GB04. Figure 7 shows the standard deviation of the mean and difference travel-time perturbations as a function of the window center and width.

It appears that at short distance $(\Delta=6.2 \mathrm{Mm})$ the travel-time definitions are very sensitive to the temporal window used, especially for the KD97 definition. The standard deviation strongly depends on this temporal window: we can almost double the noise level on the travel-time maps by simply changing the width of the window. At $6.2 \mathrm{Mm}$, the first-bounce skip on the cross-covariances is entangled at short times $[t]$ with an artifact produced by the phase-speed filter (see e.g. Couvidat and Birch, 2009), and it is entangled at larger times $[t]$ with the second-bounce skip. The artifact produced by the filter is a group of peaks at small $t$, with the overall shape of a Gabor wavelet and that partly overlap with the first-bounce skip peaks, thus affecting their shape. Therefore by shifting the center of the temporal window or by changing its width, we more or less select the parts of the first-bounce skip contaminated by this filter artifact (and to a lesser extent by the second-bounce skip). A way of alleviating this problem is by using broader phase-speed filters: the filter artifact amplitude decreases with wider filters. For a given supergranule, the value of the difference travel-time perturbation obtained with GB02 and GB04 can be larger (in absolute value) or smaller than the value obtained with the Gabor-wavelet fit depending on how we center the temporal window.

The issue with the use of the phase-speed filter and its interaction with the temporal window may potentially be discarded with the HMI data. The phase-speed filter was introduced for analysis of MDI data because of the limited spatial resolution of the short distances on the cross-covariance diagram. It is expected that the HMI optics are significantly improved. Thus we may not need to do any phase-speed filtering at short distances (for an example 

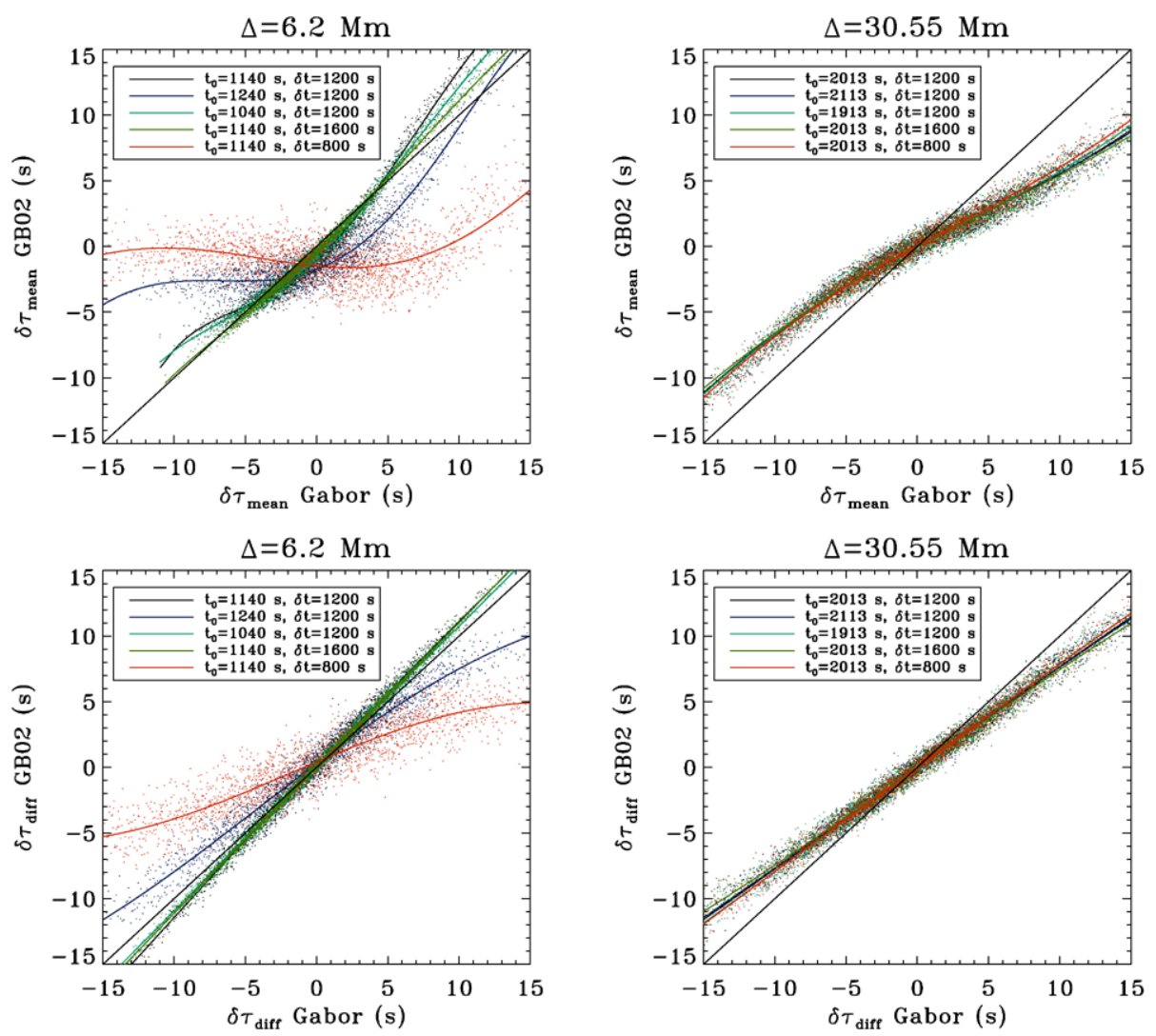

Figure 4 Scatter plots for the mean (upper panels) and difference (lower panels) travel-time perturbations obtained with the KD97 and GB02 travel-time definitions at two distances $\Delta=6.2$ and $\Delta=30.55 \mathrm{Mm}$. The thick solid lines are fifth-order polynomials fitted to the data. The different colors are for different centers $\left[t_{0}\right]$ and widths $[\delta t]$ of the temporal window used to select the first-bounce skip during the travel-time computation.

of time-distance diagram obtained with no phase-speed filters, see the one derived from Hinode data by Sekii et al., 2007). At $\Delta=30.55 \mathrm{Mm}$, the filter artifact, first-bounce and second-bounce skips, are clearly separated, explaining why the temporal window has less of an impact on the travel times.

The relationships between the KD97 travel times and GB02 or GB04 are not linear. The relationships between GB02 and GB04 are linear for small travel times ( $\leq$ five seconds in absolute value), as expected since GB04 is a linearized version of GB02.

\subsection{Active Region}

Although GB02 and GB04 were designed for quiet-Sun data, it is possible to use them for active-region data, provided that we take into account the decrease in amplitude of the crosscovariances that occurs inside sunspots (due to a reduction of the acoustic power). A simple way of taking into account this decrease is to normalize the cross-covariances $C(\Delta, \mathbf{r})-$ for a given $\Delta-$ at each location $\mathbf{r}$ by dividing $C(\Delta, \mathbf{r})$ by its maximum amplitude (L. Gizon, 

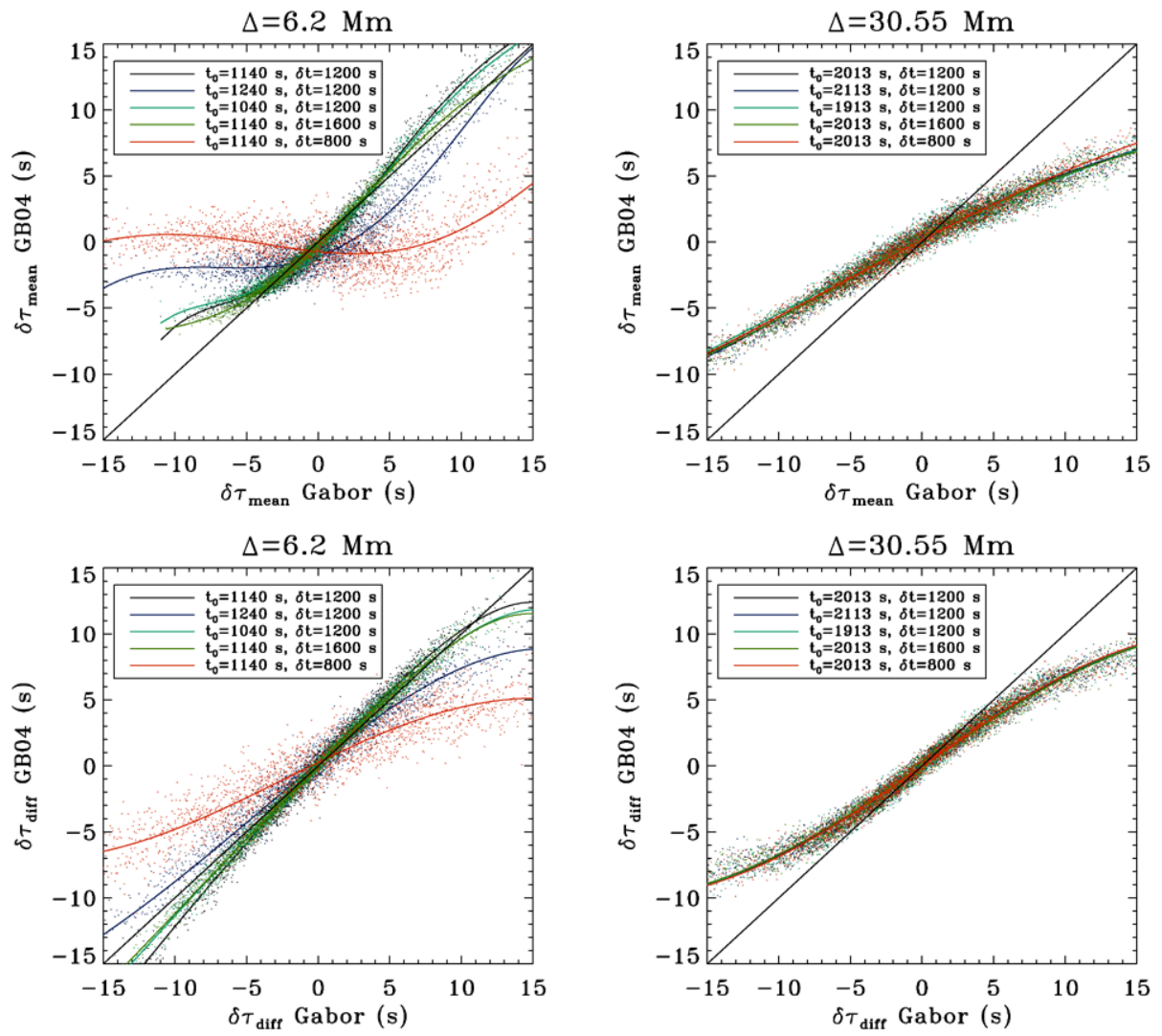

Figure 5 Scatter plots for the mean (upper panels) and difference (lower panels) travel-time perturbations obtained with the KD97 and GB04 travel-time definitions at two distances $\Delta=6.2$ and $\Delta=30.55 \mathrm{Mm}$. The thick solid lines are fifth-order polynomials fitted to the data. The different colors are for different centers $\left[t_{0}\right]$ and widths $[\delta t]$ of the temporal window used to select the first-bounce skip during the travel-time computation.

private communication, 2009): $C_{0}(\Delta, \mathbf{r})=C(\Delta, \mathbf{r}) / \max (C(\Delta, \mathbf{r}))$. GB02 and GB04 are then applied to $C_{0}(\Delta, \mathbf{r})$ instead of $C(\Delta, \mathbf{r})$. The Gabor-wavelet definition is not affected by this normalization, because it fits for the amplitude $[A]$ of the wavelet at the same time as the travel times. Note that the combination of the decrease in the amplitude of oscillations and the use of phase-speed filters may cause systematic shifts of the travel times, particularly, the mean travel times (Rajaguru et al., 2006; Parchevsky, Zhao, and Kosovichev, 2008; Nigam and Kosovichev, 2010).

For the active-region test, we use MDI Hi-Res data centered on active region NOAA 8243 of 18 June 1998. As for the quiet-Sun data cube, the line-of-sight Doppler-velocity data cube has the following dimensions: $256 \times 256 \times 512$, with a spatial resolution $\delta x=$ $\delta y=0.826 \mathrm{Mm}$, and a temporal resolution $\delta t=1$ minute. The observation duration is 512 minutes (or 8.5 hours). The reference cross-covariances $\left[C^{\text {ref }}\right]$ are obtained by spatially averaging over the ranges $x=[20,235]$ and $y=[20,80]$ in pixel units. These ranges are selected because the corresponding MDI map of the line-of-sight magnetic field shows no significant magnetic activity there. The maximum value of the cross-covariances at each 

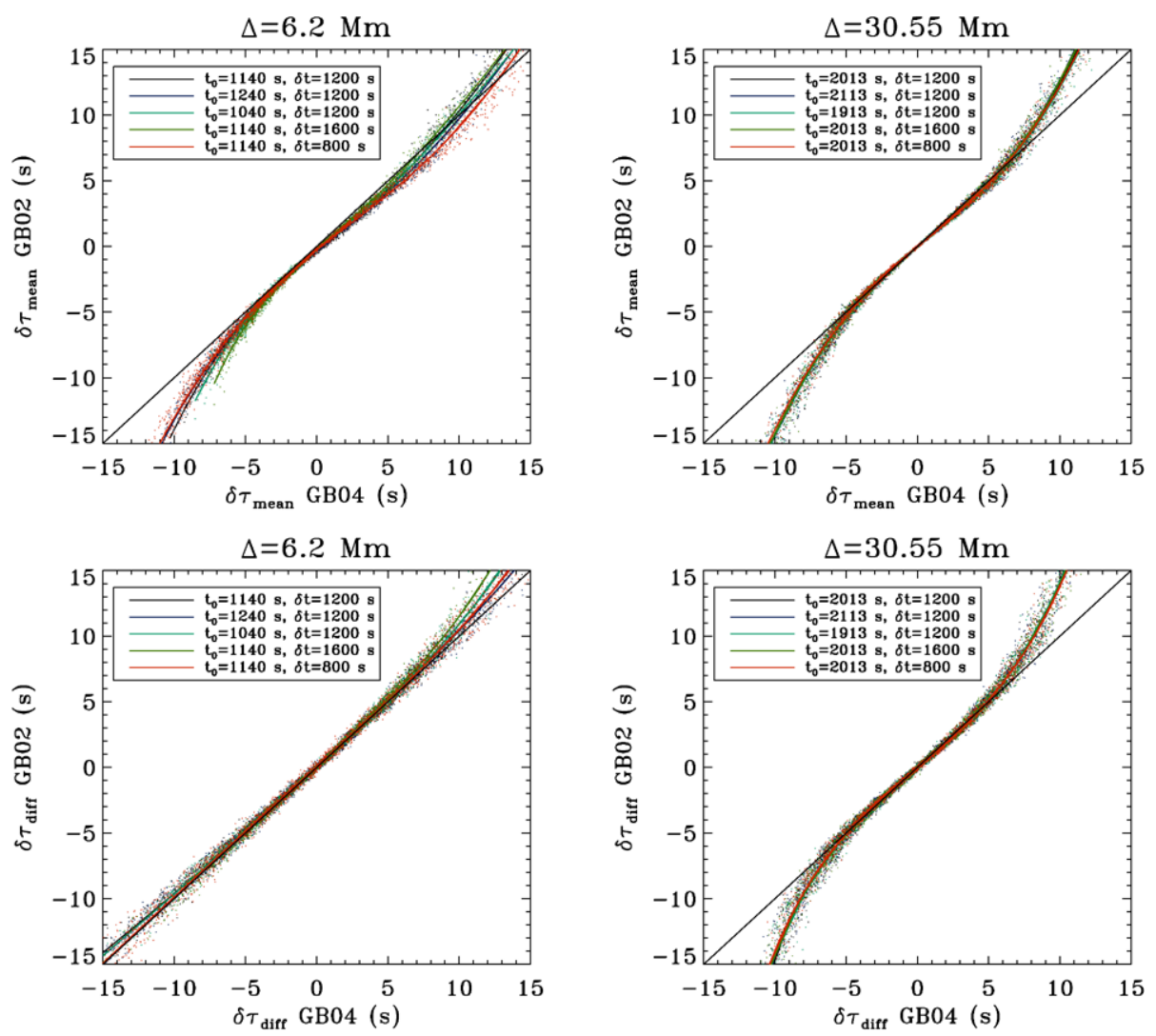

Figure 6 Scatter plots for the mean (upper panels) and difference (lower panels) travel-time perturbations obtained with the GB02 and GB04 travel-time definitions at two distances $\Delta=6.2$ and $\Delta=30.55 \mathrm{Mm}$. The thick solid lines are fifth-order polynomials fitted to the data. The different colors are for different centers $\left[t_{0}\right]$ and widths $[\delta t]$ of the temporal window used to select the first-bounce skip during the travel-time computation.

location $[\mathbf{r}]$ is obtained by Fourier interpolating these cross-covariances on a fine temporal grid and then reading their maximum amplitude. Figures 8 and 9 show the mean and difference travel-time perturbations obtained with the three definitions. The travel-time maps are rather similar especially between KD97 and GB02, showing first a positive mean traveltime perturbation at short distances $\Delta$ and then a negative perturbation. Even though KD97 and GB02 agree well with each other overall, there can be some significant discrepancies locally, in some parts of the sunspot. These discrepancies may be caused by the realization noise of solar oscillations, which may be higher in sunspots due to the smaller number of excitation events in strong field regions, and, perhaps, by some systematic effects since these travel-time definitions are not identical. The amplitude of the travel times in the sunspot is underestimated by GB04 (in absolute value) compared to the other two definitions. It appears that for sunspots, GB02 and GB04 return rather different travel-time perturbations, which can be explained by the fact that GB04 strictly reduces to GB02 only when the crosscovariances are noiseless and do not have variations in amplitude (GB04 is a linearization of GB02 with respect to $\epsilon$ for a small $\epsilon$ ), which is not the case in active regions. In addition to the strong non-linear amplitude variations of the cross-covariance in such regions, 

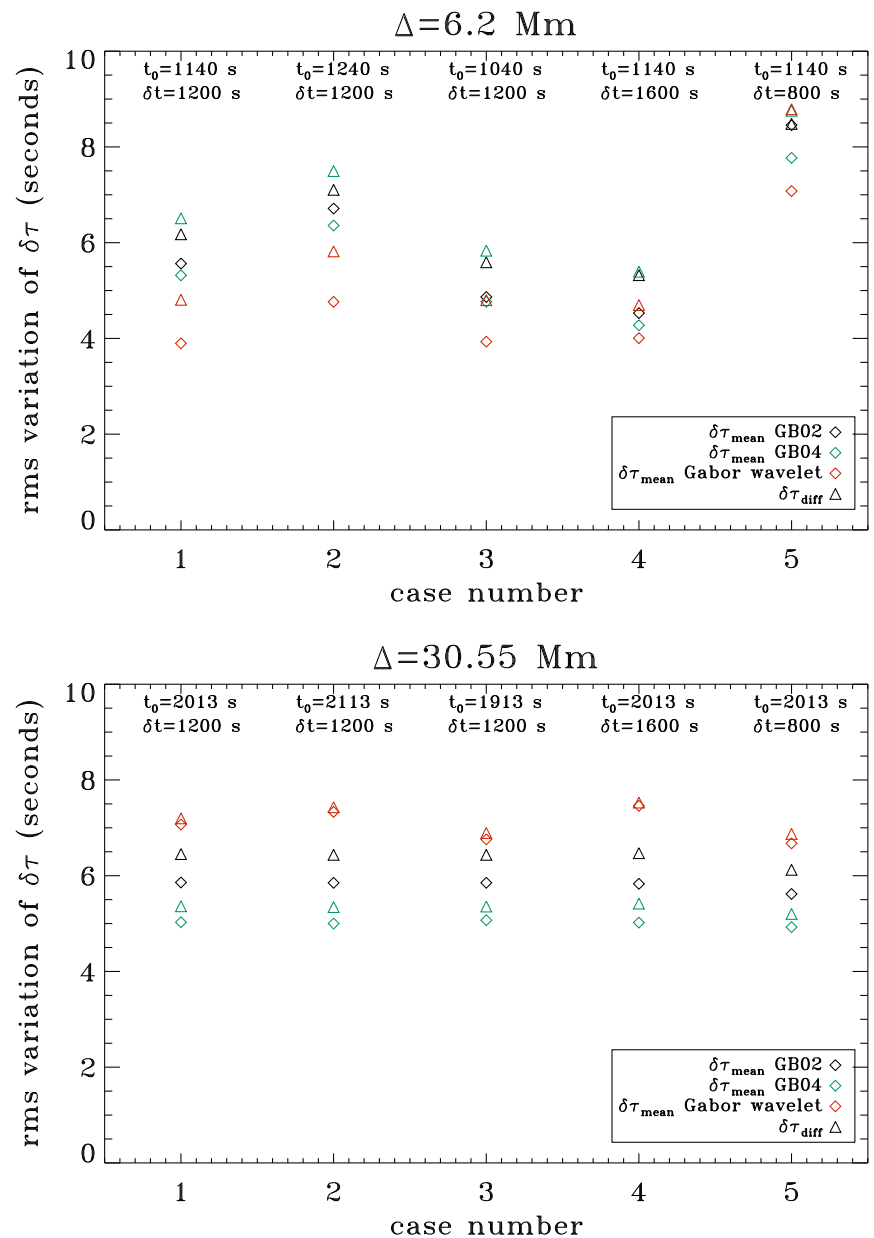

Figure 7 Standard deviations of the mean and difference travel-time perturbations at $\Delta=6.2 \mathrm{Mm}$ (upper panel), and $\Delta=30.55 \mathrm{Mm}$ (lower panel) for five different centers $\left[t_{0}\right]$ and widths $[\delta t]$ of the temporal window used to select the first-bounce ridge.

the noise level of $C(\Delta, \mathbf{r})$ is actually significantly higher than in the quiet Sun. Couvidat and Birch (2009) showed that for strong horizontal-flow velocities, the relationship between flow strength and resulting travel-time perturbation is not linear, and this non-linearity sets in much earlier (for slower flows) for GB04 than for GB02 and KD97. Therefore, we ultimately decided not to use GB04 in the pipeline. Of course, this does not preclude users of HMI data from using this or any other procedure in their work. For the linear inversions of the mean travel times in the HMI pipeline, we will use both the ray-path (Kosovichev and Duvall, 1997) and Born-approximation kernels (Birch, Kosovichev, and Duvall, 2004). Since the data-analysis pipeline will also provide the travel-time measurements described in this paper, the HMI data users will be able to develop their own inversion techniques.

Figure 10 shows the standard deviations of the mean and difference travel-time perturbations for the three travel-time definitions and for the 11 distances $[\Delta]$ studied here. These standard deviations are computed in a quiet-Sun region surrounding, but excluding, the ac- 

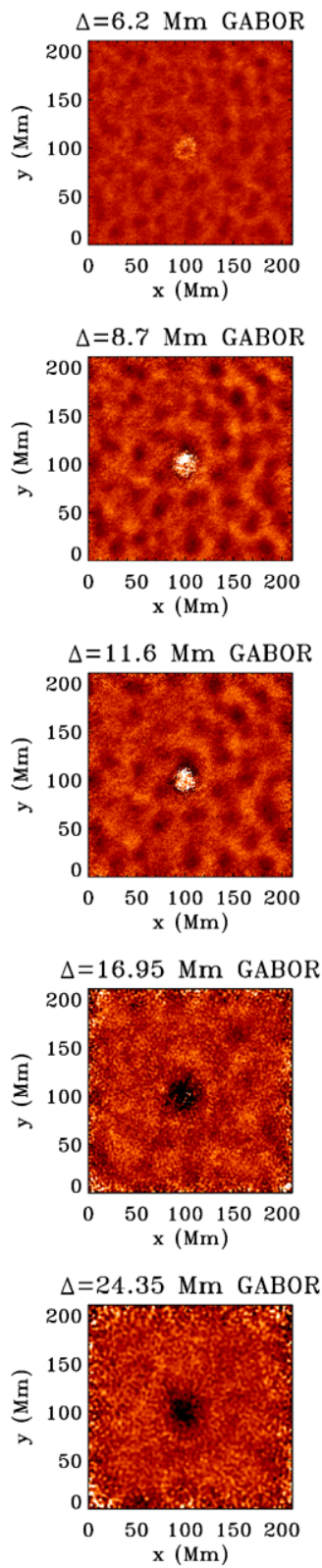
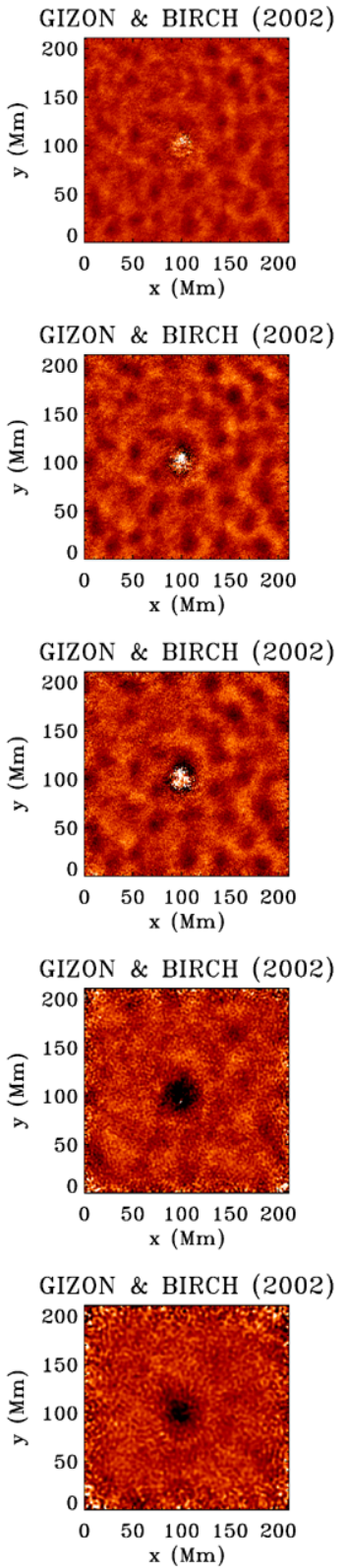
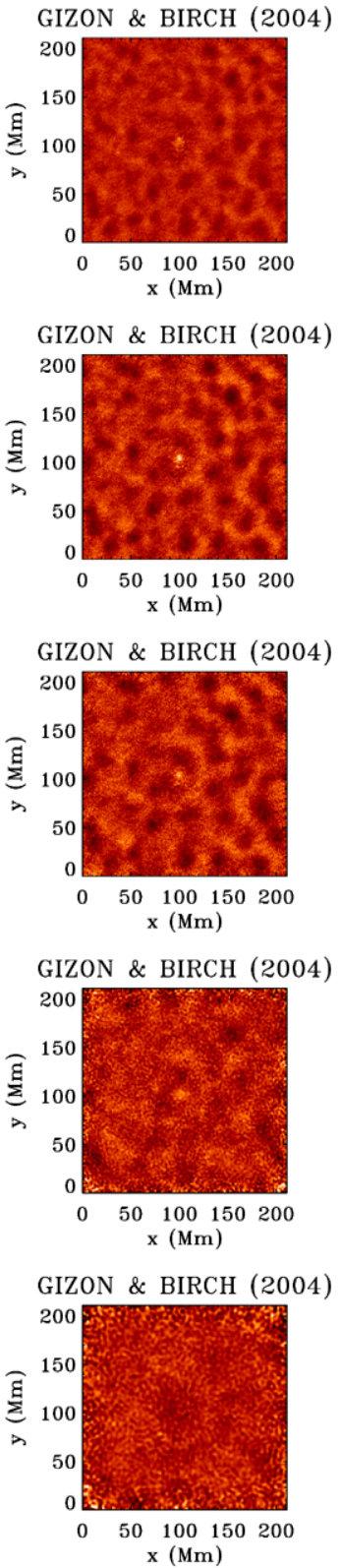

Figure 8 Difference travel-time perturbations for the active region NOAA 8243 obtained with KD97 (left column), GB02 (central column), and GB04 (right panel), and for five source-receiver distances $[\Delta]$. The colorscale is the same for all the panels, and ranges from -40 seconds to +40 seconds.

tive region. They confirm the conclusion of the previous section in which we showed that at short distances $[\Delta]$ the travel-time maps produced by the Gabor-wavelet definition have a smaller standard deviation than those produced by GB02 and GB04, while the opposite is true at larger $\Delta$. 

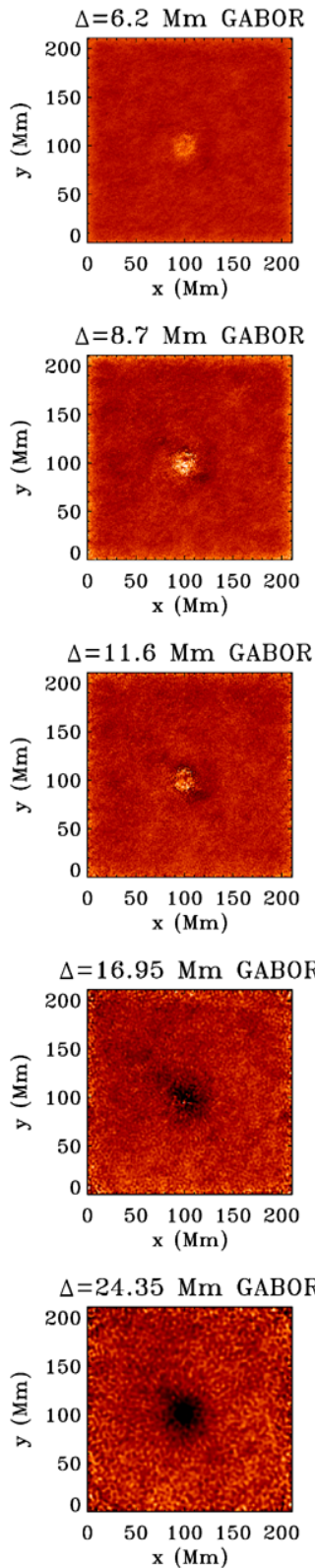
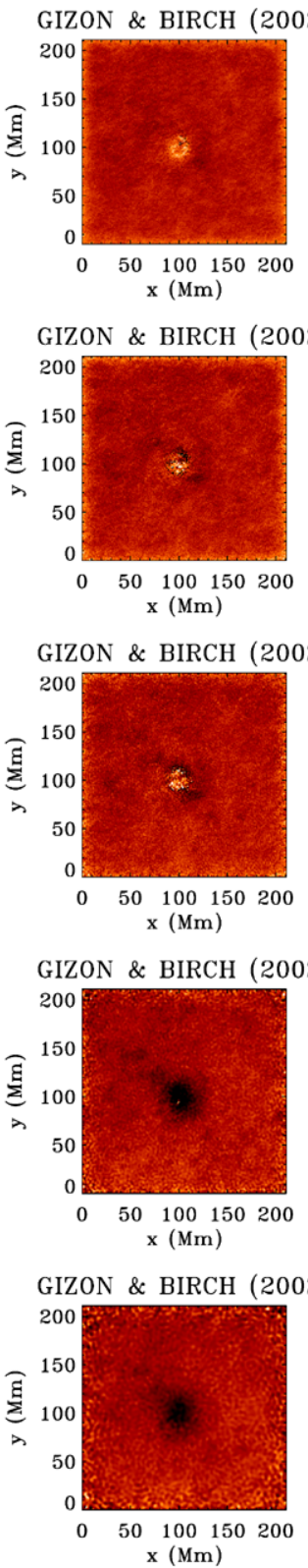

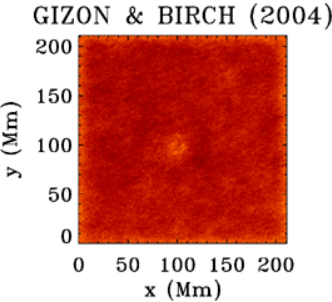

GIZON \& BIRCH (2004)

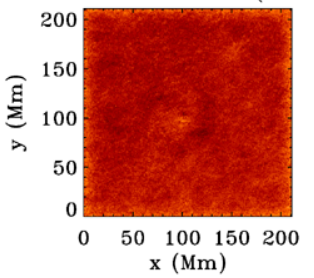

GIZON \& BIRCH (2004)
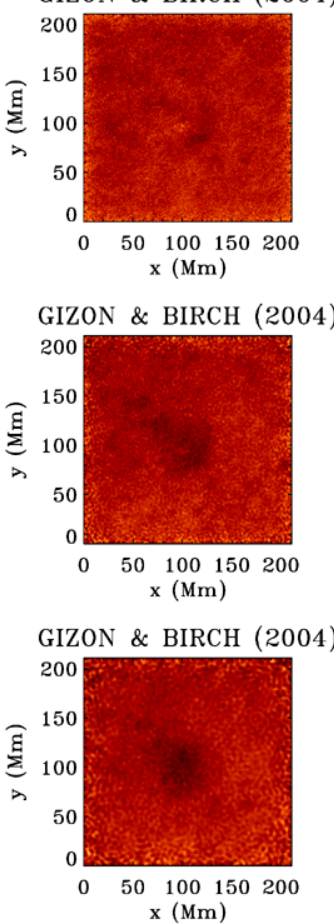

Figure 9 Mean travel-time perturbations for the active region NOAA 8243 obtained with KD97 (left column), GB02 (central column), and GB04 (right panel), and for five source-receiver distances [ $\Delta]$ ]. The colorscale is the same for all the panels, and ranges from -40 seconds to +40 seconds.

\section{Conclusion}

We have implemented three travel-time measurement procedures (Kosovichev and Duvall, 1997; Gizon and Birch 2002, 2004). The subroutines computing these travel times of 

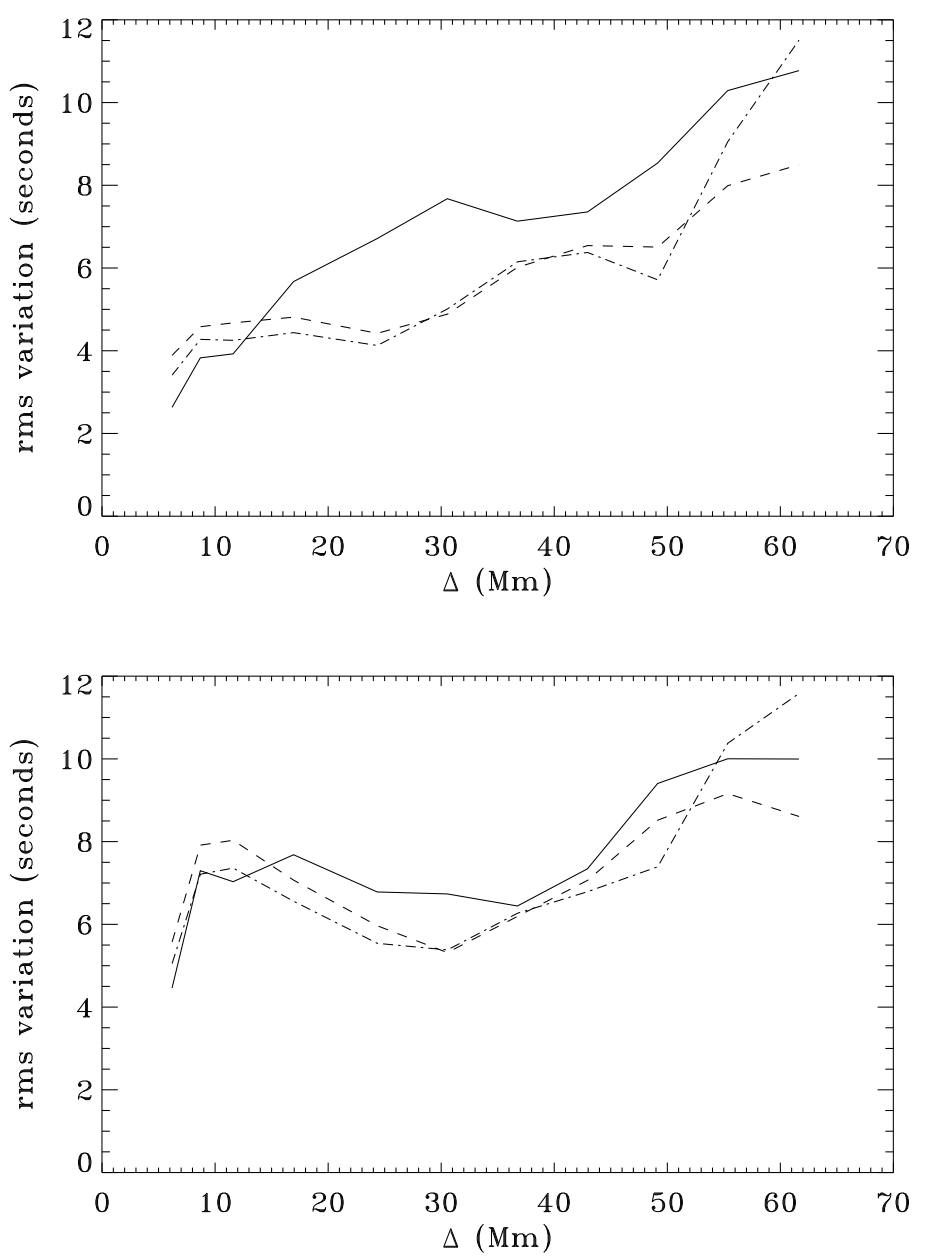

Figure 10 Upper panel: standard deviation of the mean travel-time perturbations for KD97 (solid line), GB02 (dashed line), and GB04 (dash-dotted line). Lower panel: same but for the difference travel-time perturbations.

wavepackets are part of the HMI time - distance helioseismology pipeline at the SDO Joint Science Operations Center at Stanford University. Using quiet-Sun and active-region data from the SOHO/MDI instrument we compared the mean and difference travel-time perturbations obtained by using these three definitions. They all generate similar travel times in the quiet Sun, provided that, at short distances $[\Delta]$, we use an appropriate temporal window to isolate the first-bounce skip on the cross-covariances. Indeed, at $\Delta=6.2 \mathrm{Mm}$, the travel times strongly depend on the location and width of this temporal window. This is mainly due to the phase-speed filter applied to the data cube. This phase-speed filter is used to isolate the wavefront signal from the artifact signals of the MDI instrument (Duvall et al., 1997). Without such filter, the travel-time measurements at short distances are not possible from MDI data. However, this filter introduces issues of its own: the travel times measured at short distances depend on the parameters of these phase-speed filters. Using broader filters than those considered here reduces the magnitude of this problem. HMI data might not necessitate the 
application of phase-speed filtering, which should give us access to more robust travel-time estimates at short distances. The distributions of the mean and difference travel times obtained in the quiet Sun are close to a Gaussian distribution (especially when the map edges are discarded) and are very similar for the three travel-time definitions. The Gabor-wavelet technique (Kosovichev and Duvall, 1997) produces travel-time maps with a standard deviation smaller than the Gizon and Birch $(2002,2004)$ definitions for $\Delta<15 \mathrm{Mm}$, but with a larger standard deviation for larger $\Delta$. The relationships between KD97 and GB02 or GB04 are not linear, while the relationships between GB02 and GB04 are linear for (absolute) times smaller than about five seconds. In the active region studied here, GB04 returned travel times that are significantly smaller (in absolute value) than those of GB02 and KD97. We ultimately decided not to include GB04 in the pipeline.

Acknowledgements This work was supported by NASA grant NAS5-02139 (HMI). The authors thank Laurent Gizon for suggesting a way of using the Gizon and Birch $(2002,2004)$ definitions in an active region, and for providing us with a MDI data cube of the quiet Sun.

Open Access This article is distributed under the terms of the Creative Commons Attribution Noncommercial License which permits any noncommercial use, distribution, and reproduction in any medium, provided the original author(s) and source are credited.

\section{References}

Birch, A.C., Kosovichev, A.G., Duvall, T.L. Jr.: 2004, Astrophys. J. 608, 580.

Chou, D.-Y., Duvall, T.L. Jr.: 2000, Astrophys. J. 533, 568.

Couvidat, S., Birch, A.C.: 2009, Solar Phys. 257, 217.

Couvidat, S., Birch, A.C., Kosovichev, A.G.: 2006, Astrophys. J. 640, 516.

Duvall, T.L. Jr., Gizon, L.: 2000, Solar Phys. 192, 177.

Duvall, T.L. Jr., Jefferies, S.M., Harvey, J.W., Pomerantz, M.A.: 1993, Nature 362, 430.

Duvall, T.L. Jr., D’Silva, S., Jefferies, S.M., Harvey, J.W., Schou, J.: 1996, Nature 379, 235.

Duvall, T.L. Jr., Kosovichev, A.G., Scherrer, P.H., Bogart, R.S., Bush, R.I., de Forest, C., Hoeksema, J.T., Schou, J., Saba, J.L.R., Tarbell, T.D., Title, A.M., Wolfson, C.J., Milford, P.N.: 1997, Solar Phys. 170, 63.

Gizon, L., Birch, A.C.: 2002, Astrophys. J. 571, 966.

Gizon, L., Birch, A.C.: 2004, Astrophys. J. 614, 472.

Hirzberger, J., Gizon, L., Solanki, S.K., Duvall, T.L. Jr.: 2007, Solar Phys. 251, 417.

Jackiewicz, J., Gizon, L., Birch, A.C., Duvall, T.L. Jr.: 2007, Astrophys. J. 671, 1051.

Jensen, J.M., Duvall, T.L. Jr., Jacobsen, B.H., Christensen-Dalsgaard, J.: 2001, Astrophys. J. Lett. 553, L193.

Kosovichev, A.G., Duvall, T.L. Jr.: 1997, In: Pijpers, F.P., Christensen-Dalsgaard, J., Rosenthal, C.S. (eds.) SCORe'96: Solar Convection and Oscillations and Their Relationship, Astrophys. Space Science Lib. 225, Kluwer, Dordrecht, 241.

Kosovichev, A.G., Duvall, T.L. Jr., Scherrer, P.H.: 2000, Solar Phys. 192, 159.

Lindsey, C., Braun, D.C.: 2005, Astrophys. J. Lett. 620, L1107.

Nigam, R., Kosovichev, A.G.: 2010, Astrophys. J. 708, 1475.

Parchevsky, K.V., Zhao, J., Kosovichev, A.G.: 2008, Astrophys. J. 678, 1498.

Rajaguru, S.P., Birch, A.C., Duvall, T.L. Jr., Thompson, M.J., Zhao, J.: 2006, Astrophys. J. 646, 543.

Roth, M., Gizon, L., Beck, J.G.: 2007, Astron. Nachr. 328, 215.

Scherrer, P.H., Bogart, R.S., Bush, R.I., Hoeksema, J.T., Kosovichev, A.G., Schou, J., Rosenberg, W., Springer, L., Tarbell, T.D., Title, A., Wolfson, C.J., Zayer, I., MDI Engineering Team: 1995, Solar Phys. 162, 129.

Sekii, T., Kosovichev, A.G., Zhao, J., Tsuneta, S., Shibahashi, H., Berger, T.E., Ichimoto, K., Katsukawa, Y., Lites, B., Nagata, S., Shimizu, T., Shine, R.A., Suematsu, Y., Tarbell, T.D., Title, A.M.: 2007, Publ. Astron. Soc. Japan 59, S637.

Zhao, J., Kosovichev, A.G.: 2003, In: Sawaya-Lacoste, H. (ed.) Soho 12/Gong+ 2002. Local and Global Helioseismology: The Present and Future SP-517, ESA, Noordwijk, 417.

Zhao, J., Kosovichev, A.G., Duvall, T.L. Jr.: 2001, Astrophys. J. 557, 384.

Zhao, J., Couvidat, S., Parchevsky, K.V., Bogart, R.S., Duvall, T.L. Jr., Beck, J.G., Birch, A.C., Kosovichev, A.G.: 2010, Solar Phys. submitted. 